\title{
Christlich-demokratische Wertvorstellungen im Parlamentarischen Rat 1948/49
}

\section{Von Rudolf Uertz}

\section{Christlicher Humanismus und Grundgesetz}

Ob das Grundgesetz für die Bundesrepublik Deutschland vom 23. Mai 1949 (GG) mit seinen anthropologisch-sittlichen Wertauffassungen heute noch in den Repräsentativorganen des Bundes und der Länder die erforderlichen Mehrheiten bekäme, kann bezweifelt werden. Im Europäischen Verfassungskonvent 2002/2003 jedenfalls haben laizistische Kreise gegenüber dem von der Fraktion der Europäischen Volkspartei (Christdemokraten) eingebrachten Vorschlag eines Gottesbezuges und einer Referenz an das Christentum in der Präambel entschiedenen Widerstand gezeigt, obwohl eine Präambel kein direktes Auslegungskriterium für den Verfassungstext darstellt - eine Haltung, die auch in Deutschland eine beträchtliche Anhängerschar fand.

Diese laizistische Distanzierung von religiösen und christlichen Metaphern, Bildern, Motiven und Normen im konstitutionellen, politischen und rechtlichen Raum hat tiefliegende, historisch tradierte Gründe. Im Rückblick scheint es daher als besonders beachtlich, dass das Grundgesetz für die Bundesrepublik Deutschland in so beträchtlichem Maße Grundsätze aufgenommen hat, die von der christlichen Ethik und Anthropologie beeinflusst sind. Dieses Ergebnis war nicht nur durch die Mehrheitsverhältnisse im Parlamentarischen Rat bedingt, sondern ist auch auf verschiedene andere Umstände zurückzuführen. ${ }^{1}$ Vor allem wird man nicht übersehen dürfen, dass die Hinwendung $\mathrm{zu}$ überpositiven, metaphysischen Begründungen von Grundrechten und rechtsstaatlichen Ordnungsprinzipien nach 1945 als Gegenbilder zum nationalsozialistischen Unrechtsregime und zum Rechtspositivismus weit über den kirchlich-christlichen Raum hinaus Zustimmung fand. ${ }^{2}$

1 Zum Thema vgl. Hans-Otto KleINMAnN, „Wahren, pflegen, ausbauen“ - Das Werk des Parlamentarischen Rates zwischen Grundgesetzgebung und Erneuerungsdiskurs, in: Günter Buchstab/Hans-Otto KleinmanN (Hg.), In Verantwortung vor Gott und den Menschen. Christliche Demokraten im Parlamentarischen Rat 1948/49, Freiburg i.Br. 2008, S. 14-90. - Sitzverteilung im Parlamentarischen Rat: CDU 19 Sitze, CSU 8 Sitze; SPD 27 Sitze; Liberale (FDP, LDP und DVP) 5 Sitze; Zentrum, DP und KPD je 2 Sitze.

2 In markanter Weise hat z.B. der bekannte Jurist und frühere Reichsjustizminister (SPD), Gustav RAdBruch, Die Erneuerung des Rechts, in: Werner MAIHOFER (Hg.), Naturrecht oder Positivismus?, Darmstadt 1962, S. 1ff., hier: S. 2, der selbst die längste Zeit seines Lebens Anhänger des Rechtspositivismus war, eine grundlegende Erneuerung des Rechts und der Politik auf der Basis naturrechtlicher Normen gefordert: „Die Rechtswissenschaft 
Die folgende Darstellung wird sich mit den wichtigsten Artikeln des Grundgesetzes sowie den politik- und verfassungstheoretischen Positionen der Parteien, insbesondere der CDU/CSU-Fraktion, im Parlamentarischen Rat 1948/ 49 befassen.

\section{Die Verfassungen der Länder}

Politik war im Nachkriegsdeutschland zunächst Ländersache. Die Alliierten verfolgten anfangs unterschiedliche Zielsetzungen in ihren Zonen. Vor allem die französische Besatzungsmacht hatte angesichts der Kriege mit Deutschland ein besonderes Sicherheitsstreben. Im Vergleich zu den Amerikanern und den Briten waren die Franzosen äußerst zurückhaltend, als es um Übertragung von politischen und wirtschaftlichen Kompetenzen an die Deutschen ging. Aber auch die britische Besatzungsmacht hatte es, was die Formulierung von Länderverfassungen anging, nicht eilig. Der Grund hierfür ist wohl in dem britischen Staats- und Rechtsverständnis zu suchen, das ja keine geschriebene Verfassung kennt. So wurde die Verfassung des Landes Nordrhein-Westfalen erst nach der Gründung der Bundesrepublik, nämlich im Jahre 1950, verabschiedet.

Wesentlich schneller konnten sich die Länder in der amerikanischen Zone ihre Verfassung geben: Der Freistaat Bayern sowie die Länder WürttembergBaden und Groß-Hessen verabschiedeten diese bereits im Jahr 1946, das Land Rheinland-Pfalz folgte ein Jahr später. Seine Verfassung ist neben der bayerischen die am stärksten von naturrechtlichem Gedankengut beeinflusste Konstitution. Während die Bayerische Verfassung vornehmlich von Wilhelm Hoegner, dem bekannten Staatsrechtler Hans Nawiasky sowie Hans Ehard formuliert wurde, ist das rheinland-pfälzische Verfassungsdokument weitgehend von Adolf Süsterhenn geprägt, der der naturrechtlich fundierten katholischen Staatslehre verbunden war. ${ }^{3}$ Er sollte als CDU-Vertreter auch bei der Erarbei-

muss sich wieder auf die jahrtausendalte gemeinsame Weisheit der Antike, des christlichen Mittelalters und des Zeitalters der Aufklärung besinnen, dass es ein höheres Recht gebe als das Gesetz, ein Naturrecht, ein Gottesrecht, ein Vernunftrecht, kurz ein übergesetzliches Recht, an dem gemessen das Unrecht Unrecht bleibt, auch wenn es in die Form des Gesetzes gegossen ist - vor dem auch das aufgrund eines solchen ungerechten Gesetzes gesprochene Urteil nicht Rechtsprechung ist, vielmehr Unrecht, mag auch dem Richter, eben wegen seiner positivistischen Rechtserziehung, solches Unrecht nicht zur persönlichen Schuld angerechnet werden." - Zur Problematik vgl. auch Hans MAIER, Der politische Weg der deutschen Katholiken nach 1945, in: DERS., Deutscher Katholizismus nach 1945, München 1964, S. 202; DERS., Ältere deutsche Staatslehre und westliche politische Tradition, in: DERS., Politische Wissenschaft in Deutschland, München 1969 (2. Aufl. 1985), S. 133ff., 143.

$3 \mathrm{Vgl}$. Adolf SüSTERHENN, Der Durchbruch des Naturrechts in der deutschen Verfassungsgesetzgebung nach 1945, in: Hermann CONRAD/Heinrich KIPP (Hg.), Gegenwartsprobleme des Rechts. Bd. 1: Beiträge zum Staats-, Völker- und Kirchenrecht sowie zur Rechtsphilosophie, Paderborn 1950, S. 43-52. Zu Süsterhenn vgl. R. UERTZ, Adolf Süsterhenn, in: Buchstab/KLeinMAnN, In Verantwortung vor Gott (wie Anm. 1), S. 355-364. 
tung des Bonner Grundgesetzes eine wichtige Rolle spielen. ${ }^{4}$ Einen gewissen Vorbildcharakter für die Wertauffassung des Grundgesetzes, näherhin die Menschenwürde als ,,materiellem Hauptgrundrecht“ hatte die Bayerische Verfassung. ${ }^{5}$ Dieser Sichtweise schloss sich denn auch später die Rechtsprechung des Bundesverfassungsgerichts an, das die Menschenwürde als „,tragendes Konstitutionsprinzip“ und „obersten Verfassungswert“ charakterisierte. ${ }^{6}$

Gewisse Gegenpole zu den Bayern und Rheinland-Pfalz bildeten die Verfassungen von Hessen und Bremen, die ganz und gar von säkular-liberalen und -sozialen Ideen bestimmt sind.

\section{Die Entscheidung zur Gründung der Bundesrepublik Deutschland}

Angesichts der unvereinbaren Gegensätze zwischen den USA, England und Frankreich auf der einen und der Sowjetunion auf der anderen Seite, die in ihrer Zone jegliche demokratisch-rechtsstaatlichen Bestrebungen unterdrückte, einigten sich die drei westlichen Alliierten auf der Londoner Konferenz im Juni 1948 über die zukünftigen Linien einer gemeinsamen Politik ihrer Besatzungszonen. ${ }^{7}$ Am 1. Juli 1948 wurden die Repräsentanten der westlichen Länderregierungen, die Ministerpräsidenten der Länder und die beiden Bürgermeister der Hansestädte Hamburg und Bremen, von den drei Militärgouverneuren über die Ergebnisse der Londoner Konferenz informiert. In den sogenannten Frankfurter Dokumenten empfahlen die Westmächte die Errichtung eines westdeutschen Teilstaates und die Bildung einer verfassunggebenden

4 Vgl. hierzu Burkhard van ScHEwICK, Die katholische Kirche und die Entstehung der Verfassungen in Westdeutschland 1945-1950, Mainz 1980; ferner Klaus GotTO, Die katholische Kirche und die Entstehung des Grundgesetzes, in: Anton RAUSCHER (Hg.), Kirche und Katholizismus 1945-1949, München 1977, S. 88-108; Paul MiKAT, Verfassungsziele der Kirchen unter besonderer Berücksichtigung des Grundgesetzes, in: Rudolf MORSEY/ Konrad RePgen (Hg.), Christen und Grundgesetz, Paderborn 1989, S. 33-69; aus protestantischer Sicht Axel Freiherr von CAMPENHAUSEN, Die Kirchen unter dem Grundgesetz. 1949-1989, in: EBD., S. 71-93; Gerhard BESIER, Die Rolle der Kirchen im Gründungsprozeß $\beta$ der Bundesrepublik Deutschland, Lüneburg 2000; Carsten NICOLAISEN/ Nora Andrea SchUlZE (Bearb.), Die Protokolle des Rates der Evangelischen Kirche in Deutschland, Bd. 2: 1947/48, Göttingen 1997.

5 Theodor MAUnZ, Rechtsethische Positionen in den Nachkriegsverfassungen des Bundes und der Länder; Willi GEIGER, Die Abkehr vom Rechtspositivismus in der Rechtsprechung der Nachkriegszeit 1945-1963, in: Anton RAUSCHER (Hg.), Katholizismus, Rechtsethik und Demokratiediskussion 1945-1963, Paderborn 1981, S. 9-27, 29-63.

6 Vgl. Hans Michael HeINIG, Menschenwürde, Menschenrechte, in: Evangelisches Staatslexikon, hg. von Werner Heun und Martin HONECKER, Stuttgart 2006, Sp. 1516ff., hier: 1518.

7 An der Londoner Sechsmächtekonferenz waren auch die Benelux-Staaten als direkte Nachbarn Deutschlands beteiligt. Die erste Sitzungsperiode dauerte vom 23. Februar bis 6. März, die zweite vom 20. April bis 1. Juni 1948; vgl. Gerd WeHNER, Die Westalliierten und das Grundgesetz 1948-1949, Freiburg i.Br. 1994. 
Versammlung für die Bundesrepublik Deutschland. Außerdem gab Frankreich seine Zustimmung zum Zusammenschluss seiner Besatzungszone, aus der das Saarland 1947 bis 1957 ausgegliedert und französischer Wirtschaftsverwaltung unterstellt war, mit der Bizonenverwaltung.

Im Zuge der Beschlüsse der Londoner Konferenz kam es zu zwei für die Neuordnung Westdeutschlands wichtigen Vorentscheidungen: Um ein geordnetes Wirtschafts- und Finanzsystem zu gewährleisten, wurde in den drei Westzonen am 20. Juni 1948 eine Währungsreform durchgeführt. ${ }^{8}$ Aber das im Wesentlichen von den westlichen Alliierten durchgeführte Reformwerk war nur teilweise der Grund für die - wie sich bald zeigen sollte - außerordentliche wirtschaftliche Belebung der Westzonen. Nur vier Tage später trat das sogenannte Leitsätzegesetz in Kraft, mit dem die Zwangsbewirtschaftung aufgehoben wurde, die seit der NS-Zeit bestanden hatte. ${ }^{9}$ Mit dem „Vereinigten Wirtschaftsgebiet" der zwei bzw. drei westlichen Zonen hatten die Deutschen bereits begrenzte Kompetenzen für die Verwaltung von Wirtschaft, Post und Fernmeldewesen sowie der Arbeits- und Sozialgesetzgebung. Die Entscheidung zugunsten der Liberalisierung des Marktes, die die Abgeordneten von CDU, CSU und die FDP im Frankfurter Wirtschaftsrat mitgetragen hatten, war ebenso wie die Umsetzung in erster Linie das Werk des damaligen Direktors der Wirtschaftsverwaltung, des späteren Bundeswirtschaftsministers und Bundeskanzlers Ludwig Erhard. Zu einem Zeitpunkt, da selbst noch liberale Wirtschaftswissenschaftler die Aufhebung der Zwangsbewirtschaftung als verfrüht ansahen, setzte Ludwig Erhard dieses riskante Reformwerk in die Realität um - eine Entscheidung, die bald auch erste Früchte tragen sollte und die mit die entscheidende Grundlage für den als „Wirtschaftswunder“ bezeichneten ökonomischen Wiederaufstieg Westdeutschlands bedeutete.

Mit der Einführung der „Sozialen Marktwirtschaft“ durch Ludwig Erhard - der Begriff „sozial“ intendierte die gesellschaftlich-positiven Wirkungen markt- und leistungsorientierter Ordnungsideen einerseits wie auch eine adäquate Sozialordnung und Sozialpolitik andererseits (vgl. Düsseldorfer Leitsätze der CDU vom 15. Juli 1949) - war eine wichtige Vorentscheidung auch im Hinblick auf die künftige Verfassung der Bundesrepublik Deutschland gefallen. Denn faktisch existierten mit der Installierung der Marktwirtschaft im

8 Die Französische Besatzungsmacht stimmte erst auf der Londoner Konferenz am 1. Juni 1948 dem Beitritt ihrer Zone zum Vereinigten Wirtschaftsgebiet zu.

9 Vgl. Gerold AmBrosius, Die Durchsetzung der Sozialen Marktwirtschaft in Westdeutschland 1945-1949, Stuttgart 1976; zur wirtschaftsordnungspolitischen Diskussion in der CDU vgl. Rudolf UERTZ, Christentum und Sozialismus in der frühen CDU. Grundlagen und Wirkungen der christlich-sozialen Ideen in der Union 1945-1949, Stuttgart 1981, S. 185-204; DERS., Das Ahlener Programm. Die Zonenausschusstagung der CDU der britischen Zone vom 1. bis 3. Februar 1947 und ihre Vorbereitungen, in: Die Politische Meinung 52 (2007) 446, S. 47-51. 
Vereinigten Wirtschaftsgebiet bereits wirtschafts- und gesellschaftspolitische Normen, die mittelbar auch Konsequenzen für die Verfassungsberatung des Grundgesetzes haben sollten. Jedenfalls hätte eine Aufkündigung der liberalwirtschaftlichen Ordnungsideen und ihre Ersetzung durch wirtschaftliche Lenkungsmechanismen in der neuen Bundesverfassung eigens verankert werden müssen, was jedoch nicht geschah.

Die SPD, die ebenso wie die CDU/CSU durch 27 Vertreter im Parlamentarischen Rat vertreten war, hatte zu jenem Zeitpunkt noch vor, der Bundesrepublik eine sozialistische Wirtschaftsordnung mit beträchtlichen Vollmachten für staatliche und korporative Lenkungsorgane zu geben. ${ }^{10}$ Entsprechend hätten die Sozialdemokraten alles unternehmen müssen, um ihre Ordnungsintentionen in der neuen Verfassung festzuschreiben (als Verbündete wären eigentlich nur die beiden KPD-Vertreter infrage gekommen). Doch neigten die Unionsparteien, die fünf FDP-Abgeordneten sowie DP und Zentrum mit je zwei Abgeordneten eher liberalen Ordnungsvorstellungen zu. Im Parlamentarischen Rat kündigten sich - zumindest in wichtigen Kernfragen - somit schon Koalitions- und Mehrheitsverhältnisse an, wie sie auch für den Deutschen Bundestag prägend werden sollten.

\section{Das Grundgesetz in den Beratungen des Parlamentarischen Rates}

Der Parlamentarische Rat konstituierte sich am 1. September 1948, also nur wenige Wochen nach der Londoner Konferenz. ${ }^{11}$ Sein Präsident war Konrad Adenauer. ${ }^{12}$ Vorsitzender des Hauptausschusses, in dem die Sach- und Detailfragen beraten wurden, war Carlo Schmid (SPD). Er war alles andere als ein typischer Sozialdemokrat. Der Rechtsprofessor, Schriftsteller, Übersetzer und Politiker war in der klassischen Philosophie ebenso bewandert wie in der Literatur. Eine weitere bedeutende Gestalt bei den Grundgesetzberatungen war der altliberale FDP-Politiker Professor Theodor Heuss, von 1920 bis 1933 Dozent an der Deutschen Hochschule für Politik in Berlin. Die einflussreichsten Unionsabgeordneten neben Adenauer, der als Präsident nicht mit der verfas-

10 Vgl. „Politische Leitsätze der SPD“ vom Mai 1946, in: Rainer Kunz/Herbert MAIER/ Theo Stammen, Programme der politischen Parteien in der Bundesrepublik Deutschland, Bd. 2: SPD, F.D.P., NPD, DKP, 3. Aufl., München 1979, S. 291-296; Nikolas DörR, Die Sozialdemokratische Partei Deutschlands im Parlamentarischen Rat 1948/1949. Eine Betrachtung der SPD in den Grundgesetzberatungen vor dem Hintergrund der ersten Bundestagswahl 1949, Berlin 2007.

11 Vgl. Michael F. FeldKAMP, Der Parlamentarische Rat 1948/49. Die Entstehung des Grundgesetzes, Göttingen 1998; BuCHSTAB/KLEINMANN, In Verantwortung vor Gott (wie Anm. 1).

12 Vgl. zu ihm Rudolf MORSEY, Die Rolle Konrad Adenauers im Parlamentarischen Rat, in: Vierteljahreshefte für Zeitgeschichte 18 (1970), S. 62-94; DERS., Konrad Adenauer, in: Buchstab/Kleinmann, In Verantwortung vor Gott (wie Anm. 1), S. 91-102. 
sungsrechtlichen Detailarbeit befasst war, waren der bereits erwähnte Jurist Adolf Süsterhenn, ferner der Rechtsanwalt Heinrich von Brentano (beide CDU $)^{13}$ und der Gymnasiallehrer Anton Pfeiffer (CSU). Auch die beiden letzteren hatten durch ihre zuvor erfolgten Berufungen in die Verfassunggebenden Versammlungen von Hessen bzw. Bayern einschlägige verfassungspolitische und -rechtliche Erfahrungen gesammelt. Wichtige Beiträge zu den Verfassungsberatungen lieferten ferner der Staatsrechtler Hermann von Mangoldt sowie Theophil Kaufmann (beide CDU) und Wilhelm Laforet (CSU). ${ }^{14}$

Wichtige Grundlage der Beratungen war der Entwurf des Verfassungskonvents auf Herrenchiemsee. Die Aufgabe des Herrenchiemseer Konvents (er tagte vom 10. bis 24. August 1948) hatte darin bestanden, die zentralen Verfassungsrichtlinien für das Grundgesetz zu erarbeiten. Die Unionsvertreter waren hier u.a. Süsterhenn und Pfeiffer, die zuvor auch in den beiden Verfassungsausschüssen der Union zur Vorbereitung des Grundgesetzes, dem sogenannten Heppenheimer Ausschuss und dem Ellwanger Kreises, führend beteiligt waren. ${ }^{15}$ Im Parlamentarischen Rat übernahm Pfeiffer den Vorsitz der CDU/CSU-Fraktion. ${ }^{16}$

In den Reihen der Abgeordneten des Parlamentarischen Rates war man sich weitgehend einig, dass der künftige Weststaat angesichts der Teilung Deutschlands in zwei Machtblöcke nur eine provisorische Lösung darstellen könne. Aus diesem Grunde verzichtete man auch auf den Begriff „Verfassunggebende Versammlung“"zugunsten des Begriffs „Parlamentarischer Rat“. Entsprechend

$13 \mathrm{Zu}$ Brentano vgl. Erhard H.M. LANGE, Wegbereiter der Bunderepublik. Die Abgeordneten des Parlamentarischen Rates. Brühl 1999, S. 41ff.; Manfred AGETHEN, Heinrich von Brentano, in: BuchSTAB/KLEINMANN, In Verantwortung vor Gott (wie Anm. 1), S. 123-133.

14 Vgl. Günter BuchSTAB, Theophil Kaufmann, Otto DePEnHEUER, Wilhelm Laforet, Ulrich Vosgerau, Hermann von Mangoldt, in: BuchSTAB/KLEINMANN, In Verantwortung vor Gott (wie Anm. 1), S. 193-206, 237-244, 271-282.

15 Vgl. die Grundsätze für eine deutsche Bundesverfassung. Vorschläge für die CDU/CSUArbeitsgemeinschaft, beschlossen auf der Tagung des Ellwanger Freundeskreises in Bad Brückenau am 13.4.1948. Im Ellwanger Entwurf lautet der erste Satz: „Deutschland soll ein Bundesstaat mit der Bezeichnung ,Bundesrepublik Deutschland' sein“, in: Werner SÖRGEL, Konsensus und Interessen. Eine Studie zur Entstehung des Grundgesetzes, 2. Aufl., Opladen 1985, S. 297. Zum Verfassungskonvent auf Herrenchiemsee vgl. Auf dem Weg zum Grundgesetz. Verfassungskonvent Herrenchiemsee 1948, hg. vom Haus der Bayerischen Geschichte, Augsburg 1998. Zur Entstehungsgeschichte des Grundgesetzes vgl. Hermann von MANGOLDT (Erläut.), Das Bonner Grundgesetz, Bd. 1, 2. Aufl., Berlin 1957 (Nachdr. Berlin 1966); Wolfgang BENZ (Hg.), Bewegt von der Hoffnung aller Deutschen. Zur Geschichte des Grundgesetzes, München 1979; Peter MäRZ/Heinrich OBERREUTER (Hg.), Weichenstellung für Deutschland. Der Verfassungskonvent von Herrenchiemsee, München 1999.

$16 \mathrm{Zu}$ Anton Pfeiffer vgl. Christiane Reuter, „Graue Eminenz der Bayerischen Politik“. Eine politische Biographie Anton Pfeiffers (1888-1957), München 1987; Thomas SCHLEMMER, Anton Pfeiffer, in: Buchstab/KLEINMANN, In Verantwortung vor Gott (wie Anm. 1), S. 289-298. 
sollte die staatspolitische Grundordnung der zukünftigen Bundesrepublik nicht den Namen „Verfassung“, sondern lediglich „Grundgesetz“ tragen.

\section{Provisoriumscharakter oder Vollverfassung?}

Bezüglich der Vorläufigkeit des neuen Staates und seiner Konstitution gab es zwischen den Unionsparteien und der SPD eine Kontroverse. Die Sozialdemokraten wollten aufgrund der weitreichenden alliierten Hoheitsrechte und der Vorbehalte der drei Westmächte hinsichtlich der Behandlung Deutschland als Ganzes, seiner Wiedervereinigung, der inneren und äußeren Bedrohung sowie der Stationierung alliierter Streitkräfte das Grundgesetz lediglich als „Organisationsstatut für eine Übergangszeit" verstanden wissen und alles, was in das Grundgesetz geschrieben wurde, unter das Gesetz des Provisoriums stellen. ${ }^{17}$ Die SPD wollte daher auch kein eigenes Amt für ein Staatsoberhaupt einrichten.

Die CDU/CSU konnte sich jedoch mit ihrer Vorstellung durchsetzen. So sollte das Grundgesetz ungeachtet der Tatsache, dass dieses zunächst nicht für das gesamte Deutschland gelten konnte, dennoch den Charakter einer Vollverfassung eines - wenn auch zunächst noch geteilten - souveränen Staates haben. Volle Einigkeit bestand zwischen den Parteien darin, die Grundrechte nicht in der rechtlich unbestimmten Weise der Weimarer Reichsverfassung (WRV) aufzunehmen, sondern sie stärker zu konkretisieren. Sie sollten als verbindliche Programmsätze konzipiert werden und nicht wie in der Weimarer Verfassung als „Anhängsel“ (Carlo Schmid) fungieren, wo sie hinter dem Staatsorganisationsteil platziert waren. Vielmehr sollten die Grund- und Freiheitsrechte der Person dem Verfassungswerk vorangestellt werden und als unmittelbar geltendes Recht ihre Bindewirkung auf die Verwaltung, die Rechtsprechung und die Gesetzgebung ausüben. In der Weimarer Verfassung hingegen waren die Grundrechte genau genommen Bürgerrechte, die als solche zur Disposition des Reichspräsidenten standen. ${ }^{18}$

Die der damaligen Rechtsauffassung zugrunde liegende Werthaltung ist unverkennbar ein Produkt des Rechtspositivismus, dem die meisten Berater der

17 Carlo SCHMID, Die politische und staatsrechtliche Ordnung der Bundesrepublik Deutschland, in: Die Öffentliche Verwaltung 3 (1949), S. 201-207, hier: 202, bezeichnete das westdeutsche Staatswesen als "Staatsfragment".

18 In der 3. Plenarsitzung am 9. September 1948, in: Parlamentarischer Rat. Stenographische Berichte über die Plenarsitzungen, Bonn 1948/49, S. 14, erklärte Carlo Schmidt: „Der Staat soll nicht alles tun können, was ihm gerade bequem ist, wenn er nur einen willfährigen Gesetzgeber findet, sondern der Mensch soll Rechte haben, über die auch der Staat nicht soll verfügen können. Die Grundrechte müssen das Grundgesetz regieren. ... Diese Grundrechte sollen nicht bloße Deklamationen, Deklarationen oder Direktiven sein, nicht nur Anforderungen an die Länderverfassungen, nicht nur eine Garantie der Länder-Grundrechte, sondern unmittelbar geltendes Bundesrecht, aufgrund dessen jeder einzelne Deutsche, jeder einzelne Bewohner unseres Landes vor den Gerichten soll Klage erheben können.“ 
Weimarer Reichsverfassung sowie viele Juristen und Beamte im Deutschen Reich anhingen. Da der Rechtspositivismus nicht nach der inhaltlichen Bestimmung von Recht und Gesetz fragt, sondern formale Grundsätze bei der Rechtsetzung in den Vordergrund stellt, konnte im nationalsozialistischen Staatsrecht der Führerwille als absolut gültige Rechtsquelle gerechtfertigt werden, die mit der Statuierung subjektiver Rechte unvereinbar war. ${ }^{19}$ Das führte zu der Absurdität, dass selbst die Rassegesetzgebung und die Ächtung der Juden gemäß positivistischer Rechtsauffassung den Status legaler Gesetze annehmen konnten.

\section{Besonderer Schutz der klassischen Grundrechte}

Es schien den Beratern des Grundgesetzes daher wichtig, die klassischen Grundrechte, die den einzelnen vor Übergriffen des Staates schützen sollten, verbindlich zu formulieren. Auch sollten keine Grundsätze über die soziale Lebensordnung aufgenommen werden, wie sie die Weimarer Verfassung im Abschnitt über das Wirtschaftsleben enthielt, Grundsätze die erst durch staatliche Tätigkeit hätten ausgefüllt werden müssen.

Es ist offensichtlich, dass sich die wertmäßige Verankerung des Grundgesetzes in hohem Maße aus dem Fundus naturrechtlicher und philosophischer Ideen speist. ${ }^{20}$ Der Umstand, dass die metaphysisch bzw. überpositiv begründeten Grundrechtssicherungen einschließlich der vocatio Dei in der Präambel auch von der SPD und den Vertretern der anderen Parteien mitgetragen werden konnte, zeigt an, dass laizistische Grundsätze und Einwände im Parlamentarischen Rat - abgesehen von der ohnehin wenig konstruktiven Mitarbeit der beiden KPD-Abgeordneten - kaum vertreten waren. Verständlich wird von daher ein weiteres Charakteristikum des Grundgesetzes und der Ordnung der Bundesrepublik: die besondere Betonung personalistischer und individualrechtlicher Normen bei gleichzeitiger Zurückhaltung sozial- und gesellschafts-

19 Die liberalen Grundrechte, ihre theoretische Verankerung und Bindewirkung im Grundgesetz sind diametral der nationalsozialitischen Rechts- und Staatsauffassung entgegengesetzt. Im Kommentar von Ernst Rudolf HUBER, Verfassungsrecht des Großdeutschen Reiches, 2. Aufl., Hamburg 1939, S. 361 (Auszüge in Reinhold ZIPPELIUS, Kleine deutsche Verfassungsgeschichte, München 1996, S. 137-145), heißt es: „Es gibt keine persönliche, vorstaatliche und außerstaatliche Freiheit des Einzelnen, die vom Staat zu respektieren wäre. An die Stelle des isolierten Individuums ist der in die Gemeinschaft gliedhaft eingeordnete Volksgenosse getreten, der von der Totalität des politischen Volkes erfasst und in das Gesamtwirken einbezogen ist. Es kann hier keine private staatsfreie Sphäre mehr bestehen, die der politischen Einheit gegenüber unantastbar und heilig wäre.“

20 Das Naturrecht sollte gemäß den Intentionen der meisten Vertreter des Parlamentarischen Rates nicht eine deduktive Rechtsbegründung sein; vielmehr versteht es sich gemäß dem Verständnis liberaler Staats- und Rechtstheorie als „Legitimierung, Limitierung und Normierung" des positiven Rechts (Alexander HOLLERBACH, Katholizismus und Jurisprudenz. Beiträge zur Katholizismusforschung und zur neueren Wissenschaftsgeschichte, $\mathrm{Pa}$ derborn 2004, S. 46, in Anlehnung an Eric Wolf). 
politischer Grundsätze ${ }^{21}$ - ein Umstand, der nicht zuletzt beim Vergleich von Grundgesetz und Weimarer Verfassung auffällig ist. Auch dieser Umstand ist mit Grund dafür, dass das Grundgesetz wesentlich stärker als die Reichsverfassung von 1919 beträchtliche Affinitäten zu christlich-anthropologischen Prinzipien aufweist.

Aufgrund der sozialistischen Gesellschaftsauffassung und ihrem Hang zu staatlich-administrativen Regelungen hätte die SPD zur Durchsetzung genuin sozialistischer bzw. sozialdemokratischer Positionen einen Katalog einschlägiger sozialer Grundrechte und regulierender Wirtschaftsordnungsnormen aufnehmen müssen, wie sie in nicht unerheblichem Maße auch in der Weimarer Verfassung niedergelegt worden waren. ${ }^{22}$ Dass sich die SPD aber so weitgehend mit den liberalen und liberal-christlichen Parteien auf die Beschränkung, hauptsächlich die klassischen Freiheits- und Menschenrechte aufzunehmen, festlegen ließ, lag einerseits in der schon erwähnten Einschätzung des Grundgesetzes als „Organisationsstatut für eine Übergangszeit“, andererseits in der Hoffnung, bei den Wahlen zum Ersten Deutschen Bundestag die Mehrheit zu erringen, um dann die Wirtschafts- und Sozialordnung im Sinne sozialdemokratischer Programmatik auf parlamentarisch-administrativem Weg bestimmen zu können. Schließlich befürchtete man aufgrund der Mehrheitsverhältnisse im Parlamentarischen Rat, dass die sozialen Grundrechte dort nur „verwässert" worden wären.

So hatte Kurt Schumacher, der Vorsitzende der SPD nach 1945, auf dem Parteitag der SPD am 11. September 1948 in Düsseldorf seinen Parteifreunden im Parlamentarischen Rat, dem er selbst nicht angehören wollte, die Weisung gegeben: „In Bonn werden wir Sozialdemokraten nicht den Ehrgeiz haben, die großen geschichtlichen Vorbilder der alten sozialdemokratischen Verfassungen mit Worten und Beteuerungen zu übertreffen. Hier soll ein den Realitäten entsprechendes Provisorium entstehen. "23 Auch wenn man darin übereinstimmte, dass die Grund- und Menschenrechte vor- und überstaatlichen Charakter hätten und der Parlamentarische Rat damit eine konträre Rechtsauffassung zum Gros der Abgeordneten der Weimarer Nationalversammlung einnahm, so unterschieden sich doch die Auffassungen, wie die vorstaatlichen Rechte begründet und gesichert werden sollten. ${ }^{24}$

21 Das Grundgesetz beließ es bei der sehr allgemeinen Formulierung der Sozialstaatlichkeit in den Art. 20 (1) und 28 (1).

22 Insbesondere in WRV Art. 151-165 (sogenannte Räteartikel).

23 Rede von Kurt Schumacher auf dem SPD-Parteitag 1948, in: Protokoll der Verhandlungen des Parteitages der SPD vom 11. bis 14. September 1948 in Düsseldorf, Berlin 1976, S. 44; vgl. hierzu Theo PIRKER, Die SPD nach Hitler. Die Geschichte der SPD von 19451964, München 1965, S. 88ff.

24 Zur Weimarer Reichsverfassung vgl. Gerhard ANSCHÜTZ, Die Verfassung des Deutschen Reiches vom 11. August 1919. Ein Kommentar für Wissenschaft und Praxis, 14. Aufl., Berlin 1933. 


\section{Unterschiede im Staatsverständnis}

Die diesbezüglichen Differenzen hingen eng zusammen mit dem jeweiligen Staatsverständnis. ${ }^{25}$ Während für die meisten Vertreter der SPD und FDP der Staat ein reiner Zweckverband zu sein schien, vertraten die Unionschristen und auch die Vertreter von Zentrum und DP mehrheitlich den christlich-anthropologischen bzw. naturrechtlichen Standpunkt. Adolf Süsterhenn war der bedeutendste Wortführer in den Grundsatzfragen. „Schon der große europäische Staatsphilosoph Thomas von Aquin“, so sagte er in der zweiten Plenarsitzung am 9. September 1948, ,,bekennt sich zu dem Grundsatz, dass das Volk der naturrechtliche Träger der Staatsgewalt ist, dass diese Staatsgewalt also wesenhaft beim Volke liegt." Der naturrechtlichen Staats- und Politiktheorie gemäß ist das Volk Träger der Staatsgewalt, allerdings - wie Süsterhenn hinzufügt - „im Rahmen der durch Ethik und Naturrecht gezogenen Grenzen“. Der Staat muss demnach die äußeren Voraussetzungen und Einrichtungen schaffen, ,, die es dem Menschen ermöglichen, seine körperlichen und geistigen Anlagen zu entwickeln, seine Persönlichkeit innerhalb der durch die natürlichen Sittengesetze gegebenen Schranken frei zu entfalten“. ${ }^{26}$

„Für uns“, so ergänzte der CSU-Sprecher Josef Schwalber, „ist das Volk nicht eine ungegliederte Masse, sondern ein wohlgeordneter Organismus, der sich auf den natürlichen Gemeinschaften aufbaut, auf Familie, Gemeinde und Staat." Dieser Auffassung schlossen sich die Zentrumsabgeordneten sowie die Mandatsträger der DP an, die ebenfalls naturrechtlich argumentierten. Die Freiheit des einzelnen, so äußerte der Zentrumsvertreter, ist „am sichersten und am besten gewährleistet, wenn zunächst beim Grundgesetz und später bei der Verfassung nach dem Subsidiaritätsprinzip vorgegangen wird.“27

25 Zum Staatsverständnis des Parlamentarischen Rates vgl. Werner SÖRGEL, Konsensus und Interessen. Eine Studie zur Entstehung des Grundgesetzes für die Bundesrepublik Deutschland, Stuttgart 1969, S. 188ff.

26 2. Plenarsitzung vom 8. September 1948, in: Parlamentarischer Rat (wie Anm. 18), S. 18.

27 2. Plenarsitzung vom 9. September 1948, EBD. S. 36. Zu Schwalber vgl. Oliver BRAUN, Josef Schwalber, in: BUCHSTAB/KLEINMANN, In Verantwortung vor Gott (wie Anm. 1), S. 330-340. - Volker Отто, Das Staatsverständnis des Parlamentarischen Rates. Ein Beitrag zur Entstehungsgeschichte des Grundgesetzes für die Bundesrepublik Deutschland, Düsseldorf 1971, S. 61, 198ff., vertritt die Auffassung, dass die CDU/CSU-Fraktion im Parlamentarischen Rat insgesamt den Staat im Sinne der Organismustheorie verstanden habe. Das ist so nicht zutreffend. Otto übersieht die Differenzen zwischen den klassischen Vertretern der katholischen, neuscholastisch geprägten Staatslehre einerseits und den katholischen Verfassungsrechtlern und Politikern, die mehrheitlich dem von Jacques Maritain (1882-1973; der französische Philosoph war als Vertreter Frankreichs an der Ausarbeitung der UNO-Deklaration der Menschenrechte von 1948 beteiligt) und deutschen Sozialphilosophen beeinflussten christlichen Personalismus anhingen, der sich deutlich von der neuscholastischen Variante der christlichen Staatslehre unterscheidet; vgl. Ru- 
So sehr das Grundgesetz auch subsidiäre Elemente enthält (der Begriff Subsidiarität wurde erst in einer späteren Grundgesetzänderung aufgenommen) so wenig gelang es doch den Vertretern des Naturrechts, das Subsidiaritätsprinzip ausdrücklich als Organisationsprinzip in die Verfassung aufzunehmen. Auch wenn einzelne Abgeordnete organologische Ordnungsideen mit ihrem Staats- und Gesellschaftsbild verbanden oder - wie Süsterhenn und Schwalber - Anklänge daran erkennen ließen, so hat das Grundgesetz der Vorstellung des Staates als Organismus keinen Raum gegeben. ${ }^{28}$ Vielmehr ist die Verfassung durch eine ausgesprochen personalistische Grundhaltung geprägt. Bezüglich der naturrechtlichen Herleitung der Menschenwürde einigte man sich schließlich auf die Fassung des Artikels 1: „(1) Die Würde des Menschen ist unantastbar. Sie zu achten und zu schützen ist Verpflichtung aller staatlichen Gewalt. (2) Das deutsche Volk bekennt sich darum zu unverletzlichen und unveräußerlichen Menschenrechten als Grundlage jeder menschlichen Gemeinschaft, des Friedens und der Gerechtigkeit in der Welt. (3) Die nachfolgenden Grundrechte binden Gesetzgebung, vollziehende Gewalt und Rechtsprechung als unmittelbar geltendes Recht." 29

Es folgen die Artikel des Rechts der Freiheit der Person (Art. 2), der Gleichheit vor dem Gesetz (Art. 3), der Glaubens-, Gewissens- und Bekenntnisfreiheit (Art. 4), der Meinungsfreiheit (Art. 5), der Versammlungsfreiheit (Art. 8), der Vereinigungsfreiheit (Art. 9), des Brief-, Post- und Fernmeldegeheimnisses (Art. 10), der Freizügigkeit (Art. 11), der Berufsfreiheit (Art. 12), der

dolf UERTZ, Vom Gottesrecht zum Menschenrecht. Das katholische Staatsdenken von der Französischen Revolution bis zum II. Vatikanischen Konzil (1789-1965), Paderborn 2005, S. 440ff.; Michael J. INACKER, Zwischen Transzendenz, Totalitarismus und Demokratie. Die Entwicklung des kirchlichen Demokratieverständnisses von der Weimarer Republik bis zu den Anfängen der Bundesrepublik Deutschland, Neukirchen-Vluyn 1994; vgl. auch die Interpretation des christlichen Menschenbildes des Grundgesetzes bei Günter DüRIG, Die Menschenauffassung des Grundgesetzes, in: Juristische Rundschau 7 (1952), S. 259ff.; DERS., Der Grundrechtssatz von der Menschenwürde. Entwurf eines praktikablen Wertsystems der Grundrechte, in: Archiv des öffentlichen Rechts (81) 1956, S. 117ff.; vgl. auch die Kommentierung der Art. 1 und 2 GG von Günter Dürig in: MAUNZ-DüRIG, Grundgesetz. Sonderdruck, München 2003; zur Neukommentierung von Art. 1 GG durch Matthias Herdegen (MAUNZ-DÜRIG, Grundgesetz-Kommentar, München 2003), in der Herdegen von der Position Dürigs (Kommentar von 1958: Menschenrechte als überpositive Werte) abweicht; zur Kritik vgl. Ernst-Wolfgang BöCKENFÖRDE, „Die Würde des Menschen war unantastbar. Abschied von den Verfassungsvätern. Die Neukommentierung von Artikel 1 des Grundgesetzes markiert einen Epochenbruch“, in: FAZ vom 3.9.2003.

28 Konrad Adenauer (vgl. die Grundsatzrede des Ersten Vorsitzenden der CDU für die britische Zone in der Aula der Kölner Universität vom 24.3.1946, in: Konrad ADENAUER, Reden 1917-1967. Eine Auswahl, hg. von Hans-Peter SchwARZ, Stuttgart 1975, S. 82106) vertrat unverkennbar ein personalistisches Politik- und Staatsverständnis.

29 Vgl. KleinmanN, ,Wahren, pflegen, ausbauen“ (wie Anm. 1), S. $41 \mathrm{ff}$. 
Unverletzlichkeit der Wohnung (Art. 13), daneben auch das Petitionsrecht (Art. 17).

Wenngleich das Grundgesetz - wie schon angesprochen - nicht explizit eine Wirtschaftsverfassung enthält, so zeigen doch schon die bisher aufgeführten Grundrechte, die als bürgerliche wesensmäßig zugleich auch wirtschaftliche Freiheitsrechte sind, dass im Grundgesetz eine marktwirtschaftliche Ordnung wenn auch nicht ausdrücklich formuliert, so doch sehr naheliegt, allerdings im Rahmen der noch zu betrachtenden Sozialstaatlichkeit. ${ }^{30}$ So enthält der Grundrechtsteil das (klassische) Recht auf Freiheit sowie das Eigentums- und Erbrecht (Art. 14 Abs. 1 GG). Aber im Absatz 2 dieses Artikels heißt es einschränkend: „Eigentum verpflichtet. Sein Gebrauch soll zugleich dem Wohle der Allgemeinheit dienen“. Der Eigentumsschutz gilt nicht unbedingt. Nach Abs. 3 ist nämlich eine Enteignung zum Wohle der Allgemeinheit zulässig, doch ist eine solche Maßnahme nur durch Gesetz oder aufgrund eines Gesetzes zulässig, das im Übrigen auch Art und Ausmaß der Entschädigung regelt. Und Artikel 15 sieht zum Zwecke der Vergesellschaftung die Überführung von Grund und Boden, Naturschätzen und Produktionsmitteln in Gemeineigentum oder in andere Formen der Gemeinwirtschaft vor, wobei hier wie auch in Art. 14 Abs. 3 Satz 3 und 4 die gesetzlichen Bestimmungen die Entschädigung unter Abwägung der Interessen der Allgemeinheit und der Beteiligten vorsehen. Diese Normen des Grundgesetzes in Artikel 14 und 15, wonach das Privateigentum gewährleistet wird, dieses andererseits aber (sozial) ,verpflichtet", d.h. sein Gebrauch dem Wohle der Allgemeinheit dienen soll (mit der Konsequenz, dass es gegebenenfalls auch in gemeinwirtschaftliche Formen überführt werden kann), wurde vornehmlich von der SPD vertreten. Doch sind diese Forderungen keine exklusiv sozialistisch-gewerkschaftliche Ideen, insofern die Ambivalenz von Privateigentum (privater Vorteil) und sozialem Nutzen alte Grundsätze der christlichen Sozialphilosophie sind. ${ }^{31}$

Nachdem man nicht, wie von einigen Sozialdemokraten und Gewerkschaftern gefordert, soziale Grundrechte in die Verfassung aufnehmen wollte, kam man ohne größere Diskussion überein, neben dem Prinzip der Rechtsstaatlichkeit auch das der Sozialstaatlichkeit zu unterstreichen und den rechts- und sozialstaatlichen Charakter ebenso auch für die Verfassungen der Länder bindend festzulegen. Mit der Herausstellung der Bundesrepublik als „sozialen Bundesstaat“ (Art. 20 GG) und als „sozialen Rechtsstaat“ (Art. 28 GG) do-

30 Vgl. Anm. 45.

31 Vgl. die Enzyklika Quadragesimo anno (1931), in: Texte zur katholischen Soziallehre. Die sozialen Rundschreiben der Päpste und andere kirchliche Dokumente, mit einer Einführung von Oswald von Nell-Breuning, hg. vom Bundesverband der Katholischen Arbeitnehmer-Bewegung Deutschlands, Köln 1975 (9. Aufl., Köln 2007), S. $91 \mathrm{ff}$. 
kumentiert der Parlamentarische Rat eine soziale Orientierung, ohne allerdings damit genauere instrumentelle und institutionelle Vorstellungen zu verbinden.

\section{Die kirchlichen Forderungen an den Parlamentarischen Rat}

Wie die Verbände und Gewerkschaften schalteten sich auch die Kirchen in die Beratungen des Parlamentarischen Rates ein; sie bekundeten ihr besonderes Interesse an einer grundgesetzlichen Garantie der in die christlich-abendländische Kulturordnung eingegangenen „Werte“ und „Lebensordnungen“, die Sicherung der Menschenwürde sowie der Ehe und Familie, das Erziehungsrecht der Eltern gegenüber ihren Kindern, die Unversehrtheit von Leib und Leben - auch des keimenden Lebens -, der Anerkennung des Sonntags und bestimmter kirchlicher Feiertage als Tage der Arbeitsruhe sowie die rechtliche Stellung der Kirchen.

Diesen Verfassungspostulaten wurde weitgehend Rechnung getragen. ${ }^{32}$ Vor allem Adolf Süsterhenn, der eng mit dem von Kardinal Frings für die Beobachtung der Verfassungsentwicklung beauftragten Prälaten Wilhelm Böhler zusammenarbeitete, vertrat die weitergehenden kirchlichen Forderungen bei den Beratungen. Allerdings konnten nicht alle Vorstellungen der Kirchen durchgesetzt werden; ${ }^{33}$ denn in etlichen von den beiden Kirchen vertretenen Forderungen stimmte die FDP mit der SPD. So konnte man sich auch nicht einigen, die Grundrechte als „von Gott gegeben“ zu bezeichnen bzw. ihren Ursprung in Gott bzw. im göttlichen Recht in der Verfassung zu dokumentieren. ${ }^{34}$ Man entschied sich schließlich für die Nennung Gottes in der Präambel, die die Motive, Zwecke und Absichten der Verfassunggebenden Versammlung, also deren Wertekonsens angibt, jedoch keinen verbindlichen Interpretationsrahmen für die Auslegung und Anwendung der Grundgesetzartikel bil-

32 Vgl. hierzu Gotto (wie Anm. 4); van Schewick (wie Anm. 4); SöRgEl (wie Anm. 25), S. $167-200$.

33 Die Katholische und Evangelische Kirche hatten die wichtigsten Forderungen einmütig an die Mitglieder des Parlamentarischen Rates gestellt; im Gemeinsamen Hirtenwort der Bischöfe der Bundesrepublik Deutschland vom 23. Mai 1949 hat die Katholische Kirche das Grundgesetz insgesamt gewürdigt, im Hinblick auf die nicht erfüllten Forderungen jedoch beklagt, ,dass es nicht gelungen ist, dem ganzen Grundgesetz die tiefere religiöse Begründung zu geben, um deren Verankerung christlich denkende Abgeordnete sich so sehr bemüht hatten“; in: Heinz HÜRTEN (Hg.), Katholizismus, staatliche Neuordnung und Demokratie 1945-1962, Paderborn 1991, S. 40-47, hier: 43.

34 So wandte sich Theodor Heuss gegen überzogene theologische Begründungen des Grundgesetzes, wie sie u.a. von Gerhard Kroll vertreten wurden; solche Begründungen, so gab Heuss zu bedenken, zögen nur theologische Auslegungsdebatten nach sich: „Man muss sehr vorsichtig sein um der theologischen Position willen, diese sehr diesseitigen Werke zu stark im Metaphysischen verankern zu wollen, weil man sich selber dann in eine quasi Nichtverantwortung begibt.“ 6 . Plenarsitzung am 20.10.1948, in: Parlamentarischer Rat (wie Anm. 18), S. 76. 
det. Abgelehnt wurde auch die besonders von der Katholischen Kirche nachdrücklich geforderte konfessionelle Schule, die katholische Kreise als Ausfluss des Elternrechts legitimieren wollten. ${ }^{35}$ Doch sahen trotz der Ablehnungen mancher kirchlicher Positionen die christlich-demokratischen Kräfte wie auch die Kirchen in diesem wie in den meisten anderen Artikeln des Grundgesetzes ihre religiösen und gesellschaftlich-politischen Überzeugungen weitgehend berücksichtigt.

So erklärte sich Prälat Böhler, der als Vertreter der Kirche die sicherlich weitestgehenden Forderungen gemäß der christlichen Soziallehre an das Grundgesetz stellte, in seinem „Bericht zur Frage ,Bonner Grundgesetz'“ insgesamt zufrieden und begründete dies mit dem Erreichen der Anrufung Gottes in der Präambel, der Aufnahme der allgemeinen Natur- und Menschenrechte, des besonderen Schutzes von Ehe und Familie sowie der prinzipiellen Anerkennung des Religionsunterrichts an öffentlichen Schulen.

Befriedigt äußerte sich der kirchliche Vertreter auch bezüglich des staatskirchenrechtlichen Kompromisses, d.h. die Übernahme der Kirchenartikel der Weimarer Reichsverfassung und die Annahme des Vertragsartikels, der indirekt die Bestätigung des Reichskonkordats beinhaltete. ${ }^{36}$ Auch wenn die rechtliche Stellung der Kirche nicht in den Grundrechtsteil aufgenommen wurde, konnte doch insgesamt die Position der Kirchen im künftigen Staat als akzeptabel angesehen werden. ${ }^{37}$

Nicht einverstanden zeigten sich sechs der CSU-Abgeordneten, die unter dem Hinweis auf das Subsidiaritätsprinzip der katholischen Soziallehre den föderalistischen Charakter der neuen Ordnung als nicht weitgehend genug be-

35 Vgl. aus theologischer Sicht Klaus TANNER, Gehört Gott in die Verfassung. Die Präambel des Grundgesetzes im Licht der europäischen Integration, in: Evangelische Kommentare 5 (1991), S. 260-264.

36 Es handelt sich um die aus der WRV vom 11. August 1919 übernommenen Art. 136 (Schutz der Freiheit des Bekenntnisses und der Ausübung der Religion), Art. 137 (Anerkennung des Rechtes der Kirche auf Selbstverwaltung, auf Besteuerung, auf Öffentlichkeitsrecht), Art. 138 (Schutz der Vermögensrechte), Art 139 (Sonntagsruhe), Art. 141 (Garantie der Anstaltsseelsorge).

37 GotTo (wie Anm. 4), S. 105; van Schewick (wie Anm. 4), S. 122ff., 128ff. Vgl. zum Ganzen: Der Parlamentarische Rat 1948-1949. Akten und Protokolle, hg. für den Deutschen Bundestag von Kurt Georg WernicKe, Boppard 1975ff.; Rainer SAlZMANN (Bearb.), Die CDU/CSU im Parlamentarischen Rat. Sitzungsprotokolle der Unionsfraktion (Forschungen und Quellen zur Zeitgeschichte 2), Stuttgart 1981; zu den politik- und staatstheoretischen Vorarbeiten der Unionsparteien vgl. Brigitte KAFF (Bearb.), Die Unionsparteien 1946-1950. Protokolle der Arbeitsgemeinschaft der CDU/CSU Deutschlands und der Konferenzen der Landesvorsitzenden (Forschungen und Quellen zur Zeitgeschichte 17), Düsseldorf 1991; vgl. auch Hans-Otto KLEInMAnN, Geschichte der CDU, Stuttgart 1993, S. 115ff. 
trachteten. ${ }^{38}$ Sie lehnten daher die Annahme des Grundgesetzes im Parlamentarischen Rat ebenso ab wie der Bayerische Landtag mit den Stimmen der CSU-Fraktion. ${ }^{39}$ Diese argumentierte, dass das Grundgesetz dem Bund zu viel Gewicht gebe und die Gesetzgebungskompetenzen und die Finanzhoheit der Länder über Gebühr schmälere. Doch ergänzte der Bayerische Landtag die Ablehnung durch gleichzeitiges Bekenntnis zur Bundesrepublik Deutschland und zur Verfassungstreue. Das Grundgesetz trat am 24. Mai 1949 in Kraft. ${ }^{40}$ Im Artikel 146 wird der provisorische Charakter der bundesdeutschen Konstitution hervorgehoben. Es heißt dort: „Dieses Grundgesetz verliert seine Gültigkeit an dem Tage, an dem eine Verfassung in Kraft tritt, die von dem deutschen Volke in freier Entscheidung beschlossen worden ist."

\section{Menschen- und Politikverständnis des Parlamentarischen Rates}

Das Grundgesetz hebt sich in seiner personalistischen Fundierung und seinem Freiheitsbegriff deutlich von der Programmatik der SPD der Nachkriegsphase $a b$, deren staatstheoretische Vorstellungen erst nach einem längeren politiktheoretischen und programmatischen Klärungsprozess, an dem übrigens führende katholische Sozialethiker beteiligt waren, aus den Engführungen sozialistischer Gesellschaftsanschauung befreit wurde. ${ }^{41}$ Aber schon geraume Zeit vor dem Godesberger Programm, mit dem die SPD 1959 ihre Hinwendung zu Marktwirtschaft und liberaleren Positionen einleitete, hat Carlo Schmid ein anderes Menschen- und Staatsverständnis vertreten als seine Partei. In der 9. Plenarsitzung am 6. Mai 1949 äußerte er: „Letztlich ist der Staat dazu da, die äußere Ordnung zu schaffen, deren die Menschen zu einem auf der Freiheit

38 Zum Föderalismus des Grundgesetzes und seiner ideengeschichtlichen Grundlagen vgl. Heiderose KILPER/Roland LhOTTA, Föderalismus in der Bundesrepublik Deutschland, Hagen 2004, S. 77ff.; zur föderalen Struktur des Grundgesetzes und zur Neugliederung des Bundesgebietes gemäß Verfassungsauftrag Hans-Peter SCHNEIDER (Hg.), Das Grundgesetz. Dokumentation seiner Entstehung, Bd. 9: Artikel 29 und 118 (sowie gestrichener Artikel 24 „Gebietsabtretungen“), bearb. von Carmen Abel, Frankfurt/M. 1995.

39 Der CSU-Abgeordnete Gerhard Kroll hat im Parlamentarischen Rat wie auch im Bayerischen Landtag übereinstimmend mit Prälat Georg Meixner das Grundgesetz trotz der vocatio Dei und - wie eingeräumt wurde - trotz weitgehender Zugeständnisse an christlich-sozialethische Grundsätze „letztlich als Werk des säkularisierten Geistes unseres Jahrhunderts" kritisiert. Zu Kroll vgl. Rudolf UERTZ, Konservative Kulturkritik in der frühen Bundesrepublik Deutschland. Die abendländische Akademie in Eichstätt (19521956), in: HPM 8 (2001), S. 45-71; DeRS., Gerhard Kroll, in: BuchstaB/KLEINMANN, In Verantwortung vor Gott (wie Anm. 1), S. 218-226.

40 Der Bayerische Landtag lehnte in der Sitzung am 19./20. Mai 1949 das Grundgesetz ab, konstatierte jedoch zugleich dessen Rechtsverbindlichkeit und bekundete seine Verfassungstreue.

41 Vgl. Rudolf UERTZ, Annäherungen: Christliche Sozialethik und SPD, in: HPM 13 (2006), S. $93-120$ 
des einzelnen beruhenden Zusammenlebens bedürfen. “42 Schmid hat durch seine Haltung und seine Beiträge im Parlamentarischen Rat in gewisser Weise den Weg der SPD von der marxistischen Klassenpartei zur sozialdemokratischen Volkspartei vorgezeichnet. ${ }^{43}$

Carlo Schmids Wahl in den Parlamentarischen Rat war denn auch von Unionsvertretern nicht nur ausdrücklich begrüßt, sondern vielmehr durch aktive Mithilfe gefördert worden, weil seine rechtspolitische und politiktheoretische Haltung bekannt war. So hat sich der Ministerpräsident von Württemberg-Hohenzollern, Gebhard Müller (CDU), am Arrangement mit Sozialdemokraten beteiligt, um Schmids Wahl in die Verfassunggebende Versammlung zu ermöglichen. ${ }^{44}$

Eindeutig war in dieser Hinsicht die Haltung der Freidemokraten und ihres führenden Vertreters, des späteren Bundespräsidenten Theodor Heuss. Er und seine Fraktion vertraten im wesentlichen den klassisch-liberalen Standpunkt; zwar hob sich dieser - nicht zuletzt aufgrund der Erfahrungen der Sozialen Frage des 19. Jahrhunderts und der Weltwirtschaftskrise mit ihren schlimmen Folgen - deutlich vom sogenannten Manchesterliberalismus ab und unterstützte grundsätzlich die Aufnahme des Sozialstaatsbegriffs ins Grundgesetz, doch wollte man seitens der Freien Demokraten soziale Grund- und Teilhaberechte ebenso wenig in die Verfassung aufnehmen wie die sogenannten Grundrechte der „korporativen Ordnungen“ ”zw. der intermediären Gebilde zwischen dem Einzelnen und dem Staat. Die Liberalen hielten also nach wie vor an der klassischen Gegenüberstellung von Individuum und Staat fest, wobei dem Staat die Funktion einer Ordnungsmacht zugedacht war.

Während also im liberalen Verständnis die Freiheitssicherung erste und wichtigste Aufgabe des Staates ist (der Staat setzt als Ordnungsmacht die Rahmenbedingungen für größtmögliche Freiheit, die im liberalistischen Verständnis quasi selbstregulierend auch soziale Erfolge zeitigt), setzt nach sozialdemokratischer Auffassung der Staat durch eigene Aktivitäten (umfassende Sozialrechte mit materiellen Leistungen) den einzelnen und die gesellschaftlichen Gruppen erst in die Lage, seine bzw. ihre Freiheit zu gebrauchen.

42 Parlamentarischer Rat (wie Anm. 18), S. 171.

43 Zum sozialdemokratischen Staatsverständnis vgl. Отто (wie Anm. 27), S. 203ff.; die sozialen Grundrechte, die in nicht unerheblichem Maße in der WRV enthalten waren (vgl. dort die Artikel 151-165) umfassten gemäß den Vorstellungen der SPD der Jahre 1948/ 49 u.a. die Anerkennung der Arbeit (auch als gesellschaftliche Leistung), arbeitsrechtliche Forderungen, betriebliche und überbetriebliche Mitbestimmung, Garantie des Streikrechts sowie Sicherung von Mindestlöhnen und Anspruch auf Sozialfürsorge aus öffentlichen Mitteln, ferner das Recht auf Arbeit. Die Aufnahme sozialer Grundrechte, die im Übrigen in einer Verfassung nur schwer zu fixieren sind, mindern zweifellos den Wert der „,klassischen" Grund- und Freiheitsrechte; vgl. Theodor EsCHENBURG, Geschichte der Bundesrepublik Deutschland. Bd. 1: Jahre der Besatzung 1945-1949, Stuttgart 1983, S. 490f.

44 Vgl. hierzu Salzmann (wie Anm. 37), S. XIIIf. 
Das christlich-personalistische Verständnis des Staates korrespondiert eng mit dem Subsidiaritätsprinzip. Das bedeutet, dass die Person als individuelles Handlungssubjekt und als Rechtsträger betrachtet wird, jedoch nicht im altliberalen Sinne dem Staat in jeder Beziehung bloß als Individuum gegenübersteht. Im Unterschied zur Individualität, der bloßen Vereinzelung, beinhaltet die Personalität die Sozialanlage des Menschen zu den verschiedenen Lebensund Existenzformen in Ehe, Familie, Gemeinschaft und Gesellschaft - Bereiche, die dem Staat vorgelagert sind und die - wie die individuellen Rechte und Freiheiten - ebenso unbedingt schutzwürdige Grundrechte darstellen. Zum Ausdruck kommt diese Sozialtheorie im Subsidiaritätsprinzip, das gestufte Formen der sozialen Kooperation von Einzelmensch, Gliedgemeinschaft und den ihnen - nur in bestimmter und begrenzter Hinsicht - übergeordneten staatlich-rechtlichen Institutionen intendiert.

Aus sozialdemokratischer und gewerkschaftlicher Sicht musste dieser Verzicht einen Substanzverlust bedeuten, während hingegen aus personalistischer Sichtweise das Grundgesetz dadurch mehr Konturen bekam. Beinahe wäre die Einigung auf die - wie auch immer zu interpretierende - Sozialstaatsformel durch die Unionsparteien gefährdet worden. Denn diese mussten gemäß ihrem naturrechtlich-organischen Verständnis die natürliche Ordnung der Ehe und Familie grundgesetzlich schützen; mit ihrer Position standen die Unionsparteien zwischen den Freidemokraten, die solche Forderungen grundsätzlich ablehnten und den Sozialdemokraten, die die Grundsätze der Lebensordnungen gerne im Hinblick auf die Arbeits-, Sozial- und Wirtschaftsordnung erweitert hätten. Dass sich die SPD in dieser Frage - im Widerspruch zu ihrer Sozialtheorie - bei der Forderung nach Aufnahme der sozialen Grund- und Ordnungsrechte zurückhielt, bedeutete verfassungsrechtlich und politisch gesehen eine gewisse Schwächung sozialdemokratischer Positionen im GG und entsprechend ein stärkeres Hervortreten liberaler und christlich-sozialer Grundsätze. Umgekehrt aber wurde auch die Wirtschafts- und Gesellschaftsordnung der Christdemokraten, ihr Programm der ,Sozialen Marktwirtschaft“, nicht im GG festgeschrieben. Dieses lässt diesbezüglich wohl gewisse Spielräume offen. ${ }^{45}$ Andererseits ist das Sozialstaatsprinzip, wenngleich es ein unscharfer

45 Hinsichtlich der Frage, ob die Vorschriften des Grundgesetzes eine Wirtschaftsverfassung im engeren Sinne bezeichnen, kann man drei Lehrmeinungen unterscheiden: 1. Für Herbert Krüger u.a. ist das Grundgesetz wirtschaftspolitisch neutral. 2. Eine Gruppe von Rechtswissenschaftlern (Ernst Rudolf Huber u.a.) sieht dagegen in der Sozialen Marktwirtschaft eine dem Grundgesetz adäquate Wirtschaftsordnung. 3. Hans Carl Nipperdey sieht aufgrund der Verbindung freiheitlicher und sozialer Elemente in der Sozialen Marktwirtschaft die dem Grundgesetz in besonderer Weise entsprechende Wirtschaftsordnung, ohne dass diese jedoch Alternativen ausschließe. Vgl. Heinz LAMPERT, Die Wirtschaftsund Sozialordnung der Bundesrepublik Deutschland, 12. Aufl., München 1995, S. 98ff.; Bernd RÜTHERS: Die verkannte Einheit: Staatsverfassung, Wirtschaftsverfassung, Arbeitsverfassung, Bonn 1990. 
Begriff ist, verfassungsrechtlich gesehen die wichtigste Anspruchsgrundlage für die in der prosperierenden Phase der Bundesrepublik kräftig ausgebaute Arbeits- und Sozialgesetzgebung, die spätestens seit den 1990er Jahren angesichts neuer nationaler und internationaler Herausforderungen teilweise beträchtliche Einschränkungen erfahren hat.

\section{Aporien der Weimarer Verfassung und die Reaktionen des Grundgesetzes}

Die negativen Erfahrungen mit der Weimarer Verfassung und die unbedingte Entschlossenheit, deshalb die Mängel der Verfassung von 1919 zu vermeiden, waren die feste Überzeugung der Mitglieder des Parlamentarischen Rates. Diese hatten den Willen, eine Gewaltherrschaft wie die des Nationalsozialismus künftig unmöglich zu machen. Die Willkürherrschaft des NS-Systems war durch die rechtspositivistische und obrigkeitsstaatliche Einstellung der Weimarer Verfassungsväter in erheblichem Maße begünstigt worden. Die neue Verfassung sollte, eingedenk dieses Mankos, vor- und überstaatliche Rechtsideen zum Ausgangspunkt nehmen. Diese Haltung, die Abgeordnete in allen Fraktionen vertraten, macht es unmöglich, die naturrechtlichen Ideen des GG allein oder überwiegend den Christdemokraten zuzuschreiben. ${ }^{46}$

Die Nationalsozialisten hatten - formal gesehen legal - Demokratie, Gewaltenteilung, Grundrechtsschutz und Rechtsstaatlichkeit aufgehoben. Daher sollten diese Prinzipien im Grundgesetz ausdrücklich formuliert und gegen Übergriffe von staatlich-administrativer Seite gesichert werden. Doch war die Weimarer Reichsverfassung - wenngleich sie mit Strukturdefiziten behaftet war - eine moderne liberale Verfassung, deren positive Seiten es zu berücksichtigen galt. Die Unterschiede zwischen der Reichsverfassung und dem Grundgesetz können verkürzt gesagt wie folgt beschrieben werden: ${ }^{47}$

- Die WRV spricht lediglich von einer Republik, das GG bezeichnet die Bundesrepublik als „,demokratischen Bundesstaat“ (Art. 20, 1).

- Formuliert die WRV: „Alle Staatsgewalt geht vom Volke aus“, so präzisiert das GG die Form der Demokratie, indem es ihre Ausübung durch das Volk in Wahlen und Abstimmungen sowie durch die Gewaltenteilung (Art. 20,

46 Inwieweit ins GG noch Verfassungsideen anderer Länder eingeflossen sind, ist schwer auszumachen. Jedenfalls hatte die Civil Administration Division der amerikanischen Militärregierung ein Kompendium mit dem Titel „Bundesstaatliche Verfassungen“ herausgegeben, das den Deutschen Informationsmaterial zur Erörterung der Grundsätze für eine künftige Regierung an die Hand geben sollte. In ihm waren u.a. die Verfassungen der Schweiz, Kanadas, Südafrikas, Brasiliens, der USA, der UdSSR und Italiens abgedruckt; vgl. ESCHENBURG (wie Anm. 43), S. 502.

47 Vgl. zur folgenden Gegenüberstellung die grundlegende Untersuchung von Friedrich Karl FROMME, Von der Weimarer Verfassung zum Bonner Grundgesetz. Die verfassungspolitischen Folgerungen des Parlamentarischen Rates aus Weimarer Republik und nationalsozialistischer Diktatur, (1. Aufl., Tübingen 1960) 3. Aufl., Berlin 1999, S. 197ff. 
2) genau umreißt; im Übrigen wird die Gewaltenteilung im Zusammenhang mit der Bindungswirkung der Grundrechte noch einmal ausdrücklich formuliert. In der WRV war die Gewaltenteilung - wenngleich sie die tragende Säule einer freiheitlich-demokratischen Verfassung ist - nicht eigens aufgeführt.

- Die Rechtsstaatlichkeit wird im GG nicht nur durch die vorgenannten Formulierungen gesichert, vielmehr wird als weiterer rechtsstaatlicher Grundsatz die Bindung des staatlichen Handelns an das Gesetz und die Kontrolle der Gesetzmäßigkeit des staatlichen Handelns durch die Justiz hervorgehoben.

- Sind in der WRV gemäß Artikel 48 die Grundrechte zur Disposition des Reichspräsidenten gestellt, so werden sie im GG als bindend für die drei Gewalten postuliert und darüber hinaus auch zur Fundamentalnorm der Verfassung erklärt (Art. 1); schließlich sind auch die wichtigsten demokratischen, rechtsstaatlichen und föderalen Grundentscheidung des GG und die in den Artikeln 1 und 20 niedergelegten Grundsätze einer Verfassungsänderung durch das Parlament entzogen (Art. 79,3).

- Anders als die WRV bindet das GG den Staat an Frieden und Verständigung: die ,allgemeinen Regeln des Völkerrechts“ werden zu „Bestandteilen des Bundesrechts“. Außerdem bekennt sich die Bundesrepublik zu einem System kollektiver Sicherheit und zur internationalen Schiedsgerichtsbarkeit. Die Vorbereitungen zu einem Angriffskrieg werden unter Strafe gestellt (Art. 25 u. 26).

- Das GG billigt das Widerstandrecht. Dieses erst im Zuge der sogenannten Notstandsgesetzgebung im Mai 1968 von der Großen Koalition von CDU/ CSU und SPD verabschiedete Widerstandsrecht ist jedoch insofern eingeschränkt, als es dazu berechtigt, gegen den antidemokratischen Staatsstreich, nicht jedoch gegen den demokratischen Staat vorzugehen (Art. 20, 4). ${ }^{48}$

- Die starke, noch obrigkeitsstaatliche Einstellung der Verfassungsväter der WRV kommt u.a. in deren Einstellung zu den Parteien zum Ausdruck; in der WRV werden die Parteien nur en passant angesprochen („Die Beamten sind Diener der Gesamtheit, nicht einer Partei“, WRV Art. 130, 1). Man geht 1919 noch von der „Überparteilichkeit“ einer Regierung aus, deren Herrschaft man gar als ,unpolitisch“ ansieht, insofern man die Interessen von Gruppen, Verbänden und auch der Parteien als egoistisch, nicht dem Ganzen dienend und damit eben als „unpolitisch“ abqualifiziert. Das GG

48 Dieser Artikel wurde allerdings erst bei der Grundgesetzänderung 1968 im Kontext der Diskussionen um die Notstandsgesetzgebung aufgenommen. In der Sache ist das Widerstandsrecht ein klassischer Grundsatz der Naturrechtstheorie; vgl. Karl Friedrich BERTRAM, Das Widerstandsrecht des Grundgesetzes, Berlin 1970. 
erhebt die Parteien in den Verfassungsrang, ohne ihnen allerdings den Status öffentlich-rechtlicher Körperschaften zu geben (Art. 21). ${ }^{49}$

- Im Gegensatz zur WRV ist das GG gegenüber Plebisziten misstrauisch. Der Verzicht auf unmittelbare Demokratie erfolgte aus der Überzeugung heraus, dass der Parlamentarismus nicht von außen gehemmt werden sollte. So wurde im GG das Volksvertretungsmonopol bewusst beim Parlament belassen, was die WRV nicht wollte.

- Hatte die WRV den Reichspräsidenten als einen „Hüter der Verfassung“ gedacht, als Makler zwischen den Verfassungsorganen, so hat das GG als Ausdruck effizienter Gewaltenteilung und wirksamen Verfassungsschutzes das Bundesverfassungsgericht als „Hüter der Verfassung“ etabliert, u.a. auch als „Kompetenzschlichtungsorgan“ zwischen obersten Bundesorganen (Art. 93, 1).

- Das GG will noch zwei weitere wichtige Strukturdefizite der WRV vermeiden. Die Leichtigkeit der Regierungsumstürze der Weimarer Zeit (WRV Art. 54) vermeidet das GG, indem es in Art. 67 eine Abwahl der Regierung nur dann erlaubt, wenn die abzulösende Regierung durch eine von der Parlamentsmehrheit getragene neue Regierung ersetzt wird (,konstruktives Misstrauensvotum“). Von nicht minderer Bedeutung ist auch der Grundsatz, dass die Regierung in vollem Umfange dem Parlament gegenüber verantwortlich ist. Ferner werden dem Staatsoberhaupt, dem Bundespräsidenten, nur noch unwesentliche Gestaltungsmöglichkeiten eingeräumt.

- Die WRV hat nämlich noch einen weiteren gravierenden Strukturdefekt: Wohl noch als Nachwirkungen der Monarchie und der Vorstellung, der Reichspräsident sei Nachfolger des Deutschen Kaisers (eine Art „Ersatzkaiser"), sollte er als Staatsoberhaupt unmittelbar vom Volke gewählt werden. Der Reichspräsident ist dem Parlament, dem Reichstag, nicht verantwortlich; seine Aufgabe ist vielmehr als Ersatz- und Kontrollorgan des Reichstages gedacht. Nach dem GG aber ist der Bundeskanzler gegenüber dem Reichskanzler der WRV wesentlich gestärkt worden (Kanzlerdemokratie).

- Abgeschafft ist im GG auch die Möglichkeit der unmittelbaren Mitwirkung der Länder im Bundesrat bei der Gesetzgebung, und zwar nicht nur bei der konkurrierenden, sondern auch bei der ausschließlichen Gesetzgebungskompetenz des Bundes. ${ }^{50}$

49 Vgl. zu den Parteien und zum Parteiengesetz Alf MinTZEL/Heinrich OBERREUTER (Hg.), Parteien in der Bundesrepublik Deutschland, 2. Aufl., Opladen 1992.

$50 \mathrm{Vgl}$. Rudolf Morsey, Die Entstehung des Bundesrates im Parlamentarischen Rat, in: Der Bundesrat als Verfassungsorgan und politische Kraft. Beiträge zum 25. Bestehen des Bundesrates der Bundesrepublik Deutschland, hg. vom Bundesrat, Bad Honnef 1974, S. 63-77. 
- Vor allem aber erfährt im Grundgesetz die Gewährung der Grundrechte, die in der WRV nur unzureichend geschützt, im Nationalsozialismus schließlich radikal negiert wurden, ,eine erfahrungsbedingte Ausdrücklichkeit und Intensität“" ${ }^{51}$ Der Parlamentarische Rat hält es ,nach einer Zeit, in der die Menschenwürde und die Freiheitsrechte schwer angegriffen worden waren, für notwendig, ausdrücklich zu betonen, dass ein anderer Geist dieses Verfassungswerk" beherrscht. ${ }^{52}$

\section{Schlussbetrachtung}

Das Grundgesetz der Bundesrepublik Deutschland gilt weltweit als eine der ausgewogensten und stabilsten Verfassungen. Das Verfassungswerk, das auch Ausdruck gesellschaftlich-ethischer und kulturell-geschichtlicher Erfahrungen ist, hat zweifellos einen wesentlichen Beitrag geleistet zur politisch-rechtlichen und gesellschaftlich-kulturellen Entwicklung und Stabilität der Bundesrepublik. Schließlich haben die bundesrepublikanische Verfassung und die Verfassungsrealität von Anfang an eine Faszination ausgeübt auf die Deutschen, die im Besatzungs- und Machtbereich kommunistischer Herrschaftsordnung lebten. Nach dem Zusammenbruch der kommunistischen Staaten war es daher konsequent, dass - wie im Übrigen auch beim Saarland 1957 - die Vereinigung Deutschlands am 3. Oktober 1990 durch Beitritt der DDR zum Geltungsbereich des Grundgesetzes gemäß Artikel 23 erfolgt ist. Die im Zuge der Wiedervereinigung kurz aufgeflammte Debatte um eine Neufassung des Grundgesetzes ist schnell wieder erloschen - wohl auch, weil das Grundgesetz einen verlässlichen Ordnungsrahmen für die Gegenwart und die zukünftigen Entwicklungen bietet. ${ }^{53}$

51 Fromme (wie Anm. 47), S. 198.

52 Hermann von Mangoldt in der 26. Sitzung des Hauptausschusses am 10.12.1948, in: Parlamentarischer Rat. Verhandlungen des Hauptausschusses, Bonn 1948/49, S. 306; ähnlich Adolf Süsterhenn in der 6. Plenarsitzung am 20.10.1948, in: Parlamentarischer Rat (wie Anm. 18), S. 72.

$53 \mathrm{Zu}$ den im Zuge der deutschen Einigung geführten Diskussionen über das Grundgesetz und die Ausgestaltung einer künftigen Verfassung vgl. Eine Verfassung für Deutschland, mit Beiträgen von Reinhold Zippelius, Klaus Vogel, Paul Kirchhof, Wolfhart Pannenberg, Hans-Jochen Vogel, Rupert Scholz, Max Stadler, in: Zur Debatte. Themen der Katholischen Akademie in Bayern 22 (1992), S. 1-9. 